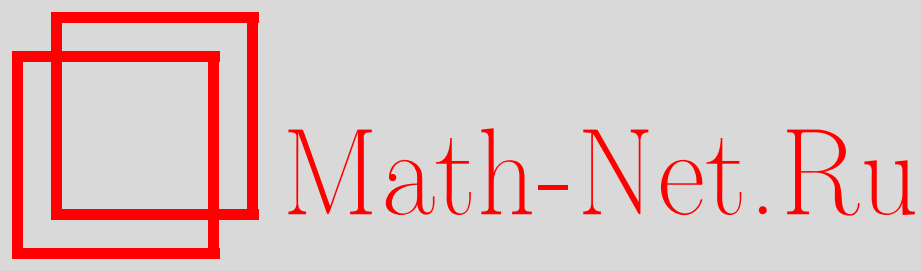

С. Г. Беневентано, И. В. Фиалковский, Е. М. Сантанжело, Нули комбинаций функций Бесселя и средний заряд графеновых наноточек, ТМФ, 2016, том 187, номер 1, 58-73

DOI: https://doi.org/10.4213/tmf8876

Использование Общероссийского математического портала Math-Net.Ru подразумевает, что вы прочитали и согласны с пользовательским соглашением http://www . mathnet.ru/rus/agreement

Параметры загрузки:

IP : 3.89.197.203

26 апреля 2023 г., $17: 43: 32$

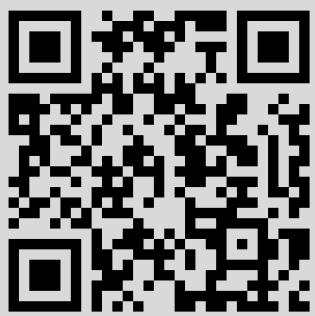




\section{НУЛИ КОМБИНАЦИЙ ФУНКЦИЙ БЕССЕЛЯ И СРЕДНИЙ ЗАРЯД ГРАФЕНОВЫХ НАНОТОЧЕК}

Установлены некоторые свойства нулей сумм и разностей последовательных функций Бесселя первого рода. При этом доказано, что нули производных функций Бесселя первого рода разных порядков упорядочены так же, как и нули самих функций Бесселя. В качестве физической мотивации рассмотрены графеновые наноточки с затвором и граничными условиями Берри-Мондрагона. Найдены разрешенные энергетические уровни и вычислен средний заряд при нулевой температуре. Подробно обсуждается его зависимость от запирающего (химического) потенциала. Также обсуждается влияние температуры.

Ключевые слова: функции Бесселя, графен, квантовые наноточки, круговые бильярды.

DOI: $10.4213 / \operatorname{tmf} 8876$

\section{1. ВВЕДЕНИЕ}

Функции Бесселя - наиболее распространенные специальные функции математической физики. Они часто появляются при решении задач с цилиндрической и сферической симметрией. В частности, при решении граничных задач, представляющих физический интерес, часто используются свойства нулей функций Бесселя, их производных, а также комбинаций различных функций Бесселя и/или их производных [1].

Некоторые свойства этих нулей известны с давних пор [2]. Другие были доказаны совсем недавно. В качестве нескольких примеров укажем на результаты работы [3], в которой были найдены некоторые ограничения, предъявляемые к первому

Работа И. В. Фиалковского поддержана FAPESP (проект 2012/22426-7). Работа С.Г. Беневентано и Е.М. Сантенжело поддержана UNLP (проект 11/X615), CONICET (PIP0681) и ANPCyT (PICT0605).

* Departamento de Física, Universidad Nacional de La Plata, Instituto de Física de La Plata, CONICET - Universidad Nacional de La Plata, La Plata, Argentina

${ }^{\dagger}$ CMCC - Universidade Federal do ABC, Santo André, S. P., Brazil

${ }^{\ddagger}$ Санкт-Петербургский государственный университет, Санкт-Петербург, Россия. E-mail: ifialk@gmail.com 
положительному нулю комбинации (включающей сам нуль) функции Бесселя и ее производной. В работе [4] изучены нули других комбинаций функций Бесселя первого рода и их производных, включая нули вторых производных как частный случай. Некоторые результаты, относящиеся к нулям функций Бесселя, представлены в работе [5]. Проблема возможного совпадения нулей функций Бесселя различных порядков, когда эти порядки отличается на величину, не являющуюся целым положительным числом, рассмотрена в работе [6]. Задача о чередовании нулей функций Бесселя первого и второго рода, а также их производных изучена в работах [7]-[9]. Наконец, в работе [10] дан анализ нулей других комбинаций функций Бесселя первого рода и их производных (а именно нулей комбинаций функций Бесселя последовательных порядков, которые снова зависят от самих нулей). Эта проблема возникает при изучении евклидовой квантовой гравитации на четырехмерном шаре.

В настоящей статье изучается относительное расположение нулей сумм и разностей функций Бесселя первого рода последовательных (действительных и неотрицательных) порядков и их чередование. В то же время мы доказываем, что правило чередования нулей производных таких функций Бесселя аналогично хорошо известному правилу чередованию нулей соответствующих функций Бесселя.

Для того чтобы физически обосновать обсуждение указанных выше линейных комбинаций функций Бесселя, мы исследуем спектр и средний заряд графеновых наноточек круговой формы, а также зависимость заряда от приложенного внешнего запирающего потенциала. В рамках континуального предела (теория Дирака) данные наноточки можно описать с помощью графенового диска с граничными условиями (ГУ) Берри-Мондрагона [11] (называемыми условиями адронного мешка [12]), наложенными на фермионы Дирака. ГУ Берри-Мондрагона являются одним из семейств ГУ, приводящих к самосопряженному гамильтониану Дирака [13], [14].

Исключительные свойства графена, представляющего собой уединенный слой атомов углерода, хорошо известны, поэтому мы не приводим здесь их подробный список. Интересующийся читатель может обратиться, например, к работам [15]-[18]. Тот факт, что графен в континуальном пределе описывается безмассовым уравнением Дирака, был теоретически обоснован в работах [19], [20] и подтвержден экспериментально более чем двадцать лет спустя в работе [21]. С тех пор получили подтверждение многие предсказания "релятивистской" безмассовой теории Дирака и теоретико-полевых методов в применении к новому материалу [22]-[24]. Среди этих предсказаний - отсутствие щели в бесконечном образце графена и неисчезающая минимальная проводимость, которые вместе представляют собой основное препятствие для дальнейшего применения этого двумерного материала в контролируемых электронных приборах. Данный факт инициировал появление огромного числа работ, нацеленных на генерацию энергетической щели в графене. Один из возможных способов достижения этой цели - использование образцов конечного размера наподобие нанолент и наноточек. Обзор достижений в этой области вместе с дополнительной литературой можно найти в нескольких недавно опубликованных обзорных статьях о переносе и электронных свойствах графеновых наноструктур, таких как наноленты и наноточки [16], [17], [25]-[27].

В предыдущей работе была изучена средняя плотность заряда и продольная проводимость разупорядоченных нанолент графена при наложении на их ребра ГУ Берри-Мондрагона [28] и проведено сравнение полученных результатов с извест- 
ными экспериментальными данными. В настоящей статье мы используем методы квантовой теории поля (КТП) для вычисления среднего заряда в графеновой наноточке с геометрией диска и выявления его зависимости от приложенного запирающего напряжения. Оказалось, что нашу модель можно применить к описанию графеновых наноточек среднего размера [29]. Предъявляемые нами расчеты показывают, что средний заряд испытывает скачки равной высоты каждый раз, когда запирающее напряжение достигает значения разрешенной собственной энергии. Поскольку эти собственные энергии определяются нулями сумм и разностей функций Бесселя первого рода последовательных целых порядков, полезно знать точно положения и правила чередования этих нулей для предсказания числа скачков в данном диапазоне запирающего напряжения.

Статья имеет следующую структуру. Мы начинаем с введения обозначений и постановки физической задачи, требующей исследования функций Бесселя - в разделе 2 представлены соглашения и обозначения и введен спектр графеновых нанодисков. Далее в разделе 3 мы формулируем и доказываем теорему 3.1 о нулях разностей функций Бесселя первого рода с различающимися на единицу порядками. Ее следствие дает точное правило чередования этих нулей. В разделе 4 мы доказываем для неотрицательных порядков теорему 4.1 об упорядочении нулей сумм тех же функций Бесселя. В качестве следствий мы получаем правило чередования этих нулей с нулями, изученными в предыдущем разделе, а также с нулями функций Бесселя и их производных. Кроме того, в этом разделе получено новое доказательство правила чередования нулей производных функций Бесселя, которое аналогично правилу чередования нулей функций Бесселя. Хотя в нашей физической задаче требуется рассматривать только функции Бесселя целых порядков, мы получаем более общий результат, справедливый для действительных неотрицательных порядков. Наконец, в разделе 5 приведен расчет среднего заряда для графеновых нанодисков Берри-Мондрагона с помощью теоретико-полевых методов [30]. Некоторые заключительные замечания и комментарии даны в разделе 6 .

\section{2. ГРАФЕНОВЫЕ НАНОДИСКИ. УСЛОВНЫЕ ОБОЗНАЧЕНИЯ И ЭНЕРГЕТИЧЕСКИЙ СПЕКТР}

При континуальном описании носителей заряда в графене, которое оказалось вполне точным [15], поведение волновой функции описывается уравнением Дирака [19], [20]. В случае одиночного конуса Дирака (т. е. долины) его можно представить в ковариантной форме

$$
\not D \psi(x)=0
$$

где $\psi=\left(\psi_{1}, \psi_{2}\right)^{T}$ - двухкомпонентный спинор,

$$
\not D=i \tilde{\gamma}^{\mu} \partial_{\mu}, \quad(\tilde{\gamma}) \equiv\left(\gamma^{0}, v_{\mathrm{F}} \gamma^{1}, v_{\mathrm{F}} \gamma^{2}\right)
$$

a $\gamma^{0}, \gamma^{1}, \gamma^{2}$ - матрицы Дирака (гамма-матрицы) размера $2 \times 2$ в одном из двух неэквивалентных представлений алгебры Клиффорда размерности $2+1$. Здесь и далее используются естественные единицы измерения, $\hbar=c=1$. Скорость Ферми $v_{\mathrm{F}}$ в этих единицах примерно равна $1 / 300$. 
В одной из двух долин выберем следующее представление матриц Дирака в пространстве-времени Минковского (с метрикой $(+,-,-))$ :

$$
\gamma^{0}=\left(\begin{array}{rr}
1 & 0 \\
0 & -1
\end{array}\right), \quad \gamma^{1}=\left(\begin{array}{rr}
0 & -1 \\
1 & 0
\end{array}\right), \quad \gamma^{2}=\left(\begin{array}{ll}
0 & i \\
i & 0
\end{array}\right) .
$$

Поскольку рассматривается четная конфигурация, вклады от разных долин и разных спинов просто суммируются, поэтому для получения результатов, соответствующих реальному образцу графена, нужно ввести коэффициент вырождения $N=4$.

Для решения поставленной задачи, т. е. задачи о диске графена радиуса $R$, наиболее подходящими являются полярные координаты $(r, \theta)$. В этих координатах соответствующий уравнению (1) гамильтониан $H$ в отсутствие внешних полей имеет вид

$$
\frac{1}{v_{\mathrm{F}}} H=i \gamma^{0} \gamma^{r} \frac{\partial}{\partial r}+\frac{i}{r} \gamma^{0} \gamma^{\theta} \frac{\partial}{\partial \theta}
$$

где

$$
\gamma^{r}=\left(\begin{array}{cc}
0 & e^{-i \theta} \\
-e^{i \theta} & 0
\end{array}\right), \quad \gamma^{\theta}=\left(\begin{array}{cc}
0 & -i e^{-i \theta} \\
-i e^{i \theta} & 0
\end{array}\right) .
$$

ГУ Берри-Мондрагона, наложенные на границах наноточки, впервые возникли при рассмотрении фермионов, локализованных в компактной области из-за наличия "запирающего" потенциала бесконечной массы [11]. Эти условия являются $(2+1)$-мерным аналогом так называемых ГУ модели адронного мешка, введенных в квантовой хромодинамике для моделирования конфайнмента в $(3+1)$-мерном пространстве [12]. Они предполагают, что ток в направлении, перпендикулярном границе, равен нулю. Записывая их в не зависящем от представления матриц $\gamma$ виде, получим

$$
\left.\frac{1+i \gamma^{\mu} n_{\mu}}{2} \psi\right|_{B}=0
$$

где $B$ - граница области, которая будет рассматриваться, а $n_{\mu}-(2+1)$-мерный вектор внешней нормали на границе. В нашем случае ГУ имеют следующий вид:

$$
\left.\frac{1+i \gamma^{r}}{2} \psi\right|_{r=R}=0
$$

Их можно также выразить через компоненты биспинора $\psi=\left(\psi_{1}, \psi_{2}\right)^{T}$ следующим образом:

$$
\psi_{1}(R, \theta)+i e^{-i \theta} \psi_{2}(R, \theta)=0 .
$$

Задачу на поиск собственных значений $H \psi=E \psi$, соответствующую гамильтониану вида (2), можно записать в явном виде следующим образом:

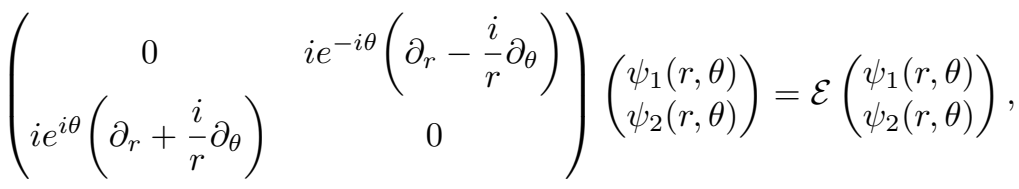

где $\mathcal{E}=E / v_{\mathrm{F}}$. 
Изучим сначала нулевые моды, т. е. случай $\mathcal{E}=0$, в котором

$$
\begin{aligned}
& \left(\partial_{r}+\frac{i}{r} \partial_{\theta}\right) \psi_{1}(r, \theta)=0 \\
& \left(\partial_{r}-\frac{i}{r} \partial_{\theta}\right) \psi_{2}(r, \theta)=0
\end{aligned}
$$

Таким образом, получим следующие однозначные решения, квадратично интегрируемые в начале координат:

$$
\psi(r, \theta)=\left(\begin{array}{c}
\sum_{n=0}^{\infty} a_{n} r^{n} e^{i n \theta} \\
\sum_{l=0}^{\infty} b_{l} r^{l} e^{-i l \theta}
\end{array}\right)
$$

где $a_{n}, b_{l}$ - некоторые произвольные постоянные. Легко проверить, что при наложении ГУ (4) нулевых мод не остается.

Если $\mathcal{E} \neq 0$, обычная процедура дает равенство

$$
\psi(r, \theta)=\left(\begin{array}{c}
\sum_{n=-\infty}^{\infty} a_{n} e^{i n \theta} J_{n}(|\mathcal{E}| r) \\
\sum_{n=-\infty}^{\infty}-i \frac{\mathcal{E} \mid}{\mathcal{E}} a_{n} e^{i(n+1) \theta} J_{n+1}(|\mathcal{E}| r)
\end{array}\right)
$$

где $J_{n}(z)$ - функция Бесселя первого рода порядка $n$, а $a_{n}$ - нормирующий множитель, который будет определен далее. Отметим, что мы уже наложили условие периодичности по $\theta$ и квадратичной интегрируемости при $r=0$. В этом случае ГУ (4) приводит к уравнениям [11], [29]

$$
\begin{aligned}
& J_{n-1}(|\mathcal{E}| R)-\frac{|\mathcal{E}|}{\mathcal{E}} J_{n}(|\mathcal{E}| R)=0, \\
& J_{n-1}(|\mathcal{E}| R)+\frac{|\mathcal{E}|}{\mathcal{E}} J_{n}(|\mathcal{E}| R)=0,
\end{aligned}
$$

Из этих уравнений видно, что спектр симметричный, поскольку уравнения в (7) меняются местами при замене $\mathcal{E} \rightarrow-\mathcal{E}$. Разрешенные моды с положительной энергией представлены выражением

$$
E_{n, k}^{\mp}=\frac{v_{\mathrm{F}}}{R} \lambda_{n-1, k}^{\mp},
$$

где $\lambda_{n-1, k}^{-}-k$-й положительный нуль разности $J_{n-1}(z)-J_{n}(z)$, a $\lambda_{n-1, k}^{+}-k$-й положительный нуль суммы $J_{n-1}(z)+J_{n}(z)$. Следовательно, нормированные собственные функции имеют вид

$$
\psi_{n, k, \alpha}^{\mp}(r, \theta)=a_{n} e^{i n \theta}\left(\begin{array}{c}
J_{n}\left(E_{n, k}^{\mp} \frac{r}{v_{\mathrm{F}}}\right) \\
\mp i \alpha e^{i \theta} J_{n+1}\left(E_{n, k}^{\mp} \frac{r}{v_{\mathrm{F}}}\right)
\end{array}\right),
$$

где $\alpha=|\mathcal{E}| / \mathcal{E}$ и

$$
a_{n}^{-2}=\frac{4 \pi^{2} R}{|\mathcal{E}|} J_{n}(|\mathcal{E}| R) J_{n+1}(|\mathcal{E}| R)
$$


Эта модель графенового диска (называемая также круговым нейтринным бильярдом) первоначально была сформулирована в работе [11]. В работе [29] показано, что предсказания этой модели довольно хорошо описывают экспериментальные результаты для графеновых наноточек диаметром $\gtrsim 100$ нм.

В двух следующих разделах мы покажем, что положительные собственные энергии можно аккуратно упорядочить и, таким образом, пронумеровать с помощью единственного индекса $q, q=1, \ldots, \infty$, так как конечной предельной точки для их последовательности не существует. Поскольку наша граничная задача является эллиптической [13], существование данной упорядоченности неудивительно, однако этот факт будет полезен при доказательстве существования энергетической щели и при оценки числа скачков среднего заряда квантовых наноточек в заданном интервале изменения запирающего напряжения.

\section{3. РАСПОЛОЖЕНИЕ ПОЛОЖИТЕЛЬНЫХ НУЛЕЙ РАЗНОСТИ $J_{\nu-1}(z)-J_{\nu}(z)$}

В этом и следующих разделах $j_{\nu, k}-k$-й положительный нуль функции $J_{\nu}(z)$, $j_{\nu, k}^{\prime}-k$-й положительный нуль функции $J_{\nu}^{\prime}(z), \lambda_{\nu-1, k}^{-}-k$-й положительный нуль разности $J_{\nu-1}(z)-J_{\nu}(z)$, где $\nu \in \mathbb{R}, \nu \geqslant 1$ и $k \in \mathbb{N} \backslash\{0\}$. Далее большинство результатов получено на основе подробного изучения хорошо известных соотношений [2], справедливых при $z>0$ :

$$
\begin{gathered}
J_{\nu-1}(z)=J_{\nu}^{\prime}(z)+\frac{\nu}{z} J_{\nu}(z) \\
J_{\nu}(z)=-J_{\nu-1}^{\prime}(z)+\frac{\nu-1}{z} J_{\nu-1}(z),
\end{gathered}
$$

и их очевидных следствий

$$
\begin{gathered}
J_{\nu-1}(z)-J_{\nu}(z)=J_{\nu}^{\prime}(z)+\frac{\nu-z}{z} J_{\nu}(z) \\
J_{\nu-1}(z)-J_{\nu}(z)=J_{\nu-1}^{\prime}(z)+\frac{z-(\nu-1)}{z} J_{\nu-1}(z) .
\end{gathered}
$$

Мы также воспользуемся столь же известным фактом, что $j_{\nu, k}>j_{\nu, k}^{\prime}>\nu$ при всех $k \geqslant 1$, и правилом чередования нулей функции $J_{\nu}(z)$.

ТеОрема 3.1. Справедливы следующие утверждения.

1. Имеет место неравенство $\lambda_{\nu-1,1}^{-}>\nu$.

2. Между двумя последовательными положительными нулями функции $J_{\nu}(z)$ существует в точности один нуль разности $J_{\nu-1}(z)-J_{\nu}(z)$, u наоборот.

3. Между двумя последовательными положительными нулями функции $J_{\nu-1}(z)$ существует в точности один нуль разности $J_{\nu-1}(z)-J_{\nu}(z)$, и наоборот.

4. Разность $J_{\nu-1}(z)-J_{\nu}(z)$ не имеет нулей в интервале $\left[j_{\nu-1, k}, j_{\nu, k}\right]$.

5. Существует в точности один положительный нуль $\lambda_{\nu-1,1}^{-}$между точками $z=0 u z=j_{\nu-1,1}, u$ этот нуль удовлетворяет условиям

$$
\begin{gathered}
j_{\nu-1,1}^{\prime}<\lambda_{\nu-1,1}^{-}<j_{\nu-1,1} \\
\nu<\lambda_{\nu-1,1}^{-}<j_{\nu, 1}^{\prime}<j_{\nu, 1}
\end{gathered}
$$


6. Для каждого $k \geqslant 1$ справедливы следующие неравенства:

$$
\begin{gathered}
j_{\nu-1, k}<j_{\nu-1, k+1}^{\prime}<\lambda_{\nu-1, k+1}^{-}<j_{\nu-1, k+1}, \\
j_{\nu, k}<\lambda_{\nu-1, k+1}^{-}<j_{\nu, k+1}^{\prime}<j_{\nu, k+1} .
\end{gathered}
$$

ДокаЗАТЕЛЬСТво. 1. В окрестности точки $z=0$

$$
J_{\nu}(z)=\left(\frac{z}{2}\right)^{\nu}\left(\frac{1}{\Gamma(\nu+1)}+\mathcal{O}\left(z^{2}\right)\right)
$$

Как следствие, для $z \neq 0$ в окрестности $z=0$ верны следующие неравенства: $J_{\nu}>0$, $J_{\nu-1}(z)-J_{\nu}(z)>0$ и $J_{\nu}^{\prime}>0$. Кроме того, известно, что $\nu<j_{\nu, 1}^{\prime}<j_{\nu, 1}$ [2]. Тогда оба члена в правой части уравнения (13) положительны при всех $0<z \leqslant \nu$ и в этом интервале нет нулей разности $J_{\nu-1}(z)-J_{\nu}(z)$. Утверждение доказано.

2. Уравнение (13) можно переписать в виде

$$
J_{\nu-1}(z)-J_{\nu}(z)=z^{-\nu} e^{z} \frac{d}{d z}\left(z^{\nu} e^{-z} J_{\nu}(z)\right) .
$$

Заметим, что между двумя нулями функции $z^{\nu} e^{-z} J_{\nu}(z)$ существует по крайней мере один нуль ее производной и, следовательно, по крайней мере один нуль разности $J_{\nu-1}(z)-J_{\nu}(z)$. Далее, нули функции $z^{\nu} e^{-z} J_{\nu}(z)$ соответствуют нулям функции $J_{\nu}(z)$, следовательно, между двумя последовательными нулями функции $J_{\nu}(z)$ есть по крайней мере один нуль разности $J_{\nu-1}(z)-J_{\nu}(z)$. Кроме того, из уравнений $(11)$ и (12) следует, что

$$
\begin{aligned}
J_{\nu-1}^{\prime}(z)-J_{\nu}^{\prime}(z) & =J_{\nu}(z)\left(\frac{\nu}{z}-1\right)+J_{\nu-1}(z)\left(\frac{\nu-1}{z}-1\right)= \\
& =J_{\nu}(z)\left(\frac{2 \nu}{z}-2-\frac{1}{z}\right)+\left(J_{\nu-1}(z)-J_{\nu}(z)\right)\left(\frac{\nu-1}{z}-1\right) .
\end{aligned}
$$

Таким образом, справедливо равенство

$$
z^{\nu-1} e^{-z} \frac{d}{d z}\left(z^{1-\nu} e^{z}\left(J_{\nu-1}(z)-J_{\nu}(z)\right)\right)=J_{\nu}(z) \frac{2 \nu-2 z-1}{z} .
$$

Последнее уравнение вместе с тем фактом, что $\lambda_{\nu-1,1}^{-}>\nu$, означает, что между двумя последовательными нулями разности $J_{\nu-1}(z)-J_{\nu}(z)$ есть по крайней мере один нуль функции $J_{\nu}(z)$. В итоге получаем, что существует единственный нуль разности $J_{\nu-1}(z)-J_{\nu}(z)$ между двумя последовательными положительными нулями функции $J_{\nu}(z)$, и наоборот. Таким образом, утверждение доказано.

3. Чтобы доказать, что между двумя последовательными положительными нулями функции $J_{\nu-1}(z)$ существует в точности один нуль разности $J_{\nu-1}(z)-J_{\nu}(z)$, и наоборот, следует воспользоваться уравнением (14), которое можно переписать в виде

$$
J_{\nu-1}(z)-J_{\nu}(z)=z^{\nu-1} e^{-z} \frac{d}{d z}\left(z^{1-\nu} e^{z} J_{\nu-1}(z)\right) .
$$

Из уравнений (11) и (12) получим также равенство

$$
z^{-\nu} e^{z} \frac{d}{d z}\left(z^{\nu} e^{-z}\left(J_{\nu-1}(z)-J_{\nu}(z)\right)\right)=J_{\nu-1}(z) \frac{2 \nu-2 z-1}{z} .
$$

Дальнейшее доказательство аналогично доказательству предыдущего пункта теоремы. Утверждение доказано. 
4. В интервале $\left[j_{\nu-1, k}, j_{\nu, k}\right]$ функции $J_{\nu-1}(z)$ и $J_{\nu}(z)$ имеют противоположные знаки. Этот факт вместе с условием $j_{\nu-1, k} \neq j_{\nu, k}$ означает, что разность $J_{\nu-1}(z)-$ $J_{\nu}(z)$ либо положительная, либо отрицательная, но никогда не равна нулю при всех $j_{\nu-1, k} \leqslant z \leqslant j_{\nu, k}$. Теперь, комбинируя утверждения пунктов 1,2 и 3 настоящей теоремы, получим, что нуль разности $J_{\nu-1}(z)-J_{\nu}(z)$, находящийся между двумя последовательными нулями функции $J_{\nu}(z)$, совпадает с нулем разности $J_{\nu-1}(z)-J_{\nu}(z)$, находящимся между двумя последовательными нулями функции $J_{\nu-1}(z)$, и лежит в интервале $\left(j_{\nu, k}, j_{\nu-1, k+1}\right)$. Утверждение доказано.

5. Как мы уже говорили выше, для $z \neq 0$ в окрестности точки $z=0$ верны неравенства $J_{\nu}>0, J_{\nu-1}(z)-J_{\nu}(z)>0$ и $J_{\nu}^{\prime}>0$. Из пункта 1 настоящей теоремы следует, что $\lambda_{\nu-1,1}^{-}>\nu>\nu-1$. Кроме того, из уравнения (14) получим, что $J_{\nu-1}(z)-J_{\nu}(z)>0$ при всех $z \in\left[\nu-1, j_{\nu-1,1}^{\prime}\right]$. Таким образом, $j_{\nu-1,1}^{\prime}<\lambda_{\nu-1,1}^{-}$. Теперь, вычисляя правую часть уравнения (14) при $z=j_{\nu-1,1}$, легко видеть, что верно неравенство $J_{\nu-1}\left(j_{\nu-1,1}\right)-J_{\nu}\left(j_{\nu-1,1}\right)<0$, а это указывает на существование по крайней мере одного нуля в интервале $j_{\nu-1,1}^{\prime}<z<j_{\nu-1,1}$. Из пункта 3 настоящей теоремы следует, что в этом интервале есть только один нуль разности $J_{\nu-1}(z)-$ $J_{\nu}(z)$, и мы получим условие (15). Далее, вычислив правую часть уравнения (13) при $z=j_{\nu, 1}^{\prime}$, заметим, что $\lambda_{\nu-1,1}^{-}<j_{\nu, 1}^{\prime}$, и, следовательно, хорошо известное свойство [2] приводит к неравенствам (16). Таким образом, утверждение доказано.

6. Еще раз исследуем поведение разности $J_{\nu-1}(z)-J_{\nu}(z)$ с помощью уравнения (14), но теперь в интервале между $j_{\nu-1, k}$ и $j_{\nu-1, k+1}$. Для определенности предположим сначала, что $J_{\nu-1}(z)>0$ в открытом интервале. Тогда в окрестности $j_{\nu-1, k}$ верно неравенство $J_{\nu-1}^{\prime}(z)>0$. Кроме того, $(z-(\nu-1)) / z>0$ в силу условий $k \geqslant 1$ и $j_{\nu-1,1}>\nu-1$ [2]. Итак, второй член в уравнении (14) всегда положителен. Что касается первого члена, он тоже положителен при $j_{\nu-1, k}<z<j_{\nu-1, k+1}^{\prime}$, поскольку $J_{\nu-1}^{\prime}(z)>0$. Как следствие, разность $J_{\nu-1}(z)-J_{\nu}(z)$ остается положительной до перехода через нуль производной, и поэтому в этом интервале нулей нет.

Далее, при $j_{\nu-1, k+1}^{\prime}<z<j_{\nu-1, k+1}$ второй член в уравнении (14) также положителен, но первый становится отрицательным и монотонно убывает. Кроме того, $J_{\nu-1}\left(j_{\nu-1, k+1}\right)-J_{\nu}\left(j_{\nu-1, k+1}\right)<0$. Согласно пункту 3 настоящей теоремы существует в точности один нуль разности между $j_{\nu-1, k}$ и $j_{\nu-1, k+1}$ и он таков, что верна цепочка неравенств $j_{\nu-1, k}<j_{\nu-1, k+1}^{\prime}<\lambda_{\nu-1, k+1}^{-}<j_{\nu-1, k+1}$, как указано в уравнении (17). Легко проверить, что тот же вывод следует из предположения, что $J_{\nu-1}(z)<0$ в открытом интервале.

Наконец, аналогичный анализ знаков разности $J_{\nu-1}(z)-J_{\nu}(z)$ с помощью уравнения (13) приводит к соотношению (18). Утверждение и теорема доказаны.

СледСтвиЕ 3.1. Справедливы следующие неравенства:

$$
\lambda_{\nu, k+1}^{-}<\lambda_{\nu-1, k+2}^{-}<\lambda_{\nu, k+2}^{-} .
$$

ДокАЗАтЕЛьСтво. Проведя в (17) замену $\nu \rightarrow \nu+1$, получим неравенства

$$
j_{\nu, k}<j_{\nu, k+1}^{\prime}<\lambda_{\nu, k+1}^{-}<j_{\nu, k+1} .
$$

Подставляя последнее полученное неравенство в неравенство (18), в котором заменим $k$ на $k+1$, и опять используя (17), но на этот раз заменяя $k$ на $k+1$ и $\nu$ на $\nu+1$, завершаем доказательство правила чередования нулей разности $J_{\nu-1}(z)-J_{\nu}(z)$. Утверждение доказано. 


\section{4. РАСПОЛОЖЕНИЕ ПОЛОЖИТЕЛЬНЫХ НУЛЕЙ СУММЫ $J_{\nu-1}(z)+J_{\nu}(z)$}

В этом разделе $j_{\nu, k}-k$-й положительный нуль функции $J_{\nu}(z), j_{\nu, k}^{\prime}-k$-й положительный нуль функции $J_{\nu}^{\prime}(z), \lambda_{\nu-1, k}^{+}-k$-й положительный нуль суммы $J_{\nu-1}(z)+$ $J_{\nu}(z)$, где $\nu \in \mathbb{R}, \nu \geqslant 1$ и $k \in \mathbb{N} \backslash\{0\}$. Бо́льшая часть нижеследующих результатов получена в результате изучения хорошо известных соотношений [2], справедливых при $z>0$ :

$$
\begin{gathered}
J_{\nu-1}(z)+J_{\nu}(z)=J_{\nu}^{\prime}(z)+\frac{\nu+z}{z} J_{\nu}(z) \\
J_{\nu-1}(z)+J_{\nu}(z)=-J_{\nu-1}^{\prime}(z)+\frac{z+(\nu-1)}{z} J_{\nu-1}(z) .
\end{gathered}
$$

Теорема 4.1. Справедливы следующие утверждения.

1. В интервале $0<z \leqslant j_{\nu-1,1}$ нет положительных нулей суммы $J_{\nu-1}(z)+J_{\nu}(z)$.

2. Между двумя последовательными нулями функции $J_{\nu}(z)$ есть в точности один нуль суммы $J_{\nu-1}(z)+J_{\nu}(z)$, и наоборот.

3. Между двумя последовательными нулями функиии $J_{\nu-1}(z)$ есть в точности один нуль суммы $J_{\nu-1}(z)+J_{\nu}(z)$, и наоборот.

4. В интервале между точками $z=0 u z=j_{\nu, 1}$ есть в точности один положительный нуль функиии $\lambda_{\nu-1,1}^{+}$, причем он удовлетворяет условию

$$
j_{\nu, 1}^{\prime}<\lambda_{\nu-1,1}^{+}<j_{\nu, 1}
$$

5. Сумма $J_{\nu-1}(z)+J_{\nu}(z)$ не имеет нулей в интервале $\left[j_{\nu, k}, j_{\nu-1, k+1}\right]$.

6. Для всех $k \geqslant 1$ верны неравенства

$$
\begin{gathered}
j_{\nu, k}<j_{\nu, k+1}^{\prime}<\lambda_{\nu-1, k+1}^{+}<j_{\nu, k+1}, \\
j_{\nu-1, k+1}<\lambda_{\nu-1, k+1}^{+}<j_{\nu-1, k+2}^{\prime}<j_{\nu-1, k+2} .
\end{gathered}
$$

ДоказАтельство. 1 . В интервале $\left(0, j_{\nu-1,1}\right)$ обе функции $J_{\nu-1}$ и $J_{\nu}$ положительны. В самом деле, как мы уже упоминали в начале теоремы 3.1, они обе положительны в окрестности начала координат, поскольку $z \neq 0$. Кроме того, первый нуль функции $J_{\nu}$ больше $j_{\nu-1,1}$ [2]. Таким образом, утверждение доказано.

2. Уравнение (21) можно записать в виде

$$
J_{\nu-1}(z)+J_{\nu}(z)=z^{-\nu} e^{-z} \frac{d}{d z}\left(z^{\nu} e^{z} J_{\nu}(z)\right) .
$$

Следовательно, между двумя последовательными нулями функции $J_{\nu}(z)$ есть по крайней мере один нуль суммы $J_{\nu-1}(z)+J_{\nu}(z)$. Из уравнений $(11)$ и (12) следует равенство

$$
z^{\nu-1} e^{z} \frac{d}{d z}\left(z^{1-\nu} e^{-z}\left(J_{\nu-1}(z)+J_{\nu}(z)\right)\right)=-J_{\nu}(z) \frac{2 \nu+2 z-1}{z}
$$

которое вместе с фактом, что $2 \nu+2 z-1>0$ при всех $\nu \geqslant 1$, означает, что между двумя последовательными нулями суммы $J_{\nu-1}(z)+J_{\nu}(z)$ есть по крайней мере 
один нуль функции $J_{\nu}(z)$. В итоге получим, что между двумя последовательными положительными нулями функции $J_{\nu}(z)$ существует единственный нуль суммы $J_{\nu-1}(z)+J_{\nu}(z)$, и наоборот. Утверждение доказано.

3. Чтобы доказать, что между двумя последовательными положительными нулями функции $J_{\nu-1}(z)$ есть в точности один нуль суммы $J_{\nu-1}(z)+J_{\nu}(z)$, и наоборот, следует воспользоваться уравнением (22), которое можно переписать в виде

$$
J_{\nu-1}(z)+J_{\nu}(z)=-z^{\nu-1} e^{z} \frac{d}{d z}\left(z^{1-\nu} e^{-z} J_{\nu-1}(z)\right) .
$$

Кроме того, из уравнений (11) и (12) следует равенство

$$
z^{-\nu} e^{-z} \frac{d}{d z}\left(z^{\nu} e^{z}\left(J_{\nu-1}(z)+J_{\nu}(z)\right)\right)=J_{\nu-1}(z) \frac{2 \nu+2 z-1}{z} .
$$

Дальнейшее доказательство аналогично доказательству предыдущего пункта теоремы. Таким образом, утверждение доказано.

4. Как уже упоминалось, при $z \neq 0$ в окрестности $z=0$ справедливы неравенства $J_{\nu}>0, J_{\nu-1}(z)+J_{\nu}(z)>0$ и $J_{\nu}^{\prime}>0$. Из правой части уравнения $(21)$ получим неравенства $J_{\nu-1}(z)+J_{\nu}(z)>0$ для всех $z \in\left(0, j_{\nu, 1}^{\prime}\right]$. Далее, вычисляя правую часть уравнения (21) при $z=j_{\nu, 1}$, легко заметить, что $J_{\nu-1}\left(j_{\nu, 1}\right)+J_{\nu}\left(j_{\nu, 1}\right)<0$, а это указывает на то, что в интервале $j_{\nu, 1}^{\prime}<z<j_{\nu, 1}$ есть по крайней мере один нуль. В силу пункта 2 настоящей теоремы существует только один нуль суммы $J_{\nu-1}(z)+J_{\nu}(z)$ в этом интервале, и мы получаем, что верно условие (23). Утверждение доказано.

5 . В интервале $\left(j_{\nu, k}, j_{\nu-1, k+1}\right)$ функции $J_{\nu-1}(z)$ и $J_{\nu}(z)$ имеют одинаковые знаки. Вместе с условием $j_{\nu-1, k+1} \neq j_{\nu, k}$ это означает, что сумма $J_{\nu-1}(z)+J_{\nu}(z)$ либо положительна, либо отрицательна, но не равна нулю при всех $j_{\nu, k} \leqslant z \leqslant j_{\nu-1, k+1}$. Из утверждений пунктов $1-4$ настоящей теоремы следует, что нуль $\lambda_{\nu-1, k+1}^{+}$суммы $J_{\nu-1}(z)+J_{\nu}(z)$ в интервале между $j_{\nu-1, k+1}$ и $j_{\nu-1, k+2}$ совпадает с нулем суммы $J_{\nu-1}(z)+J_{\nu}(z)$ в интервале между $j_{\nu, k}$ и $j_{\nu, k+1}$ и лежит в интервале $\left(j_{\nu-1, k+1}, j_{\nu, k+1}\right)$. Таким образом, утверждение доказано.

6. Еще раз исследуем поведение суммы $J_{\nu-1}(z)+J_{\nu}(z)$ с помощью уравнения $(22)$, но на этот раз в интервале между $j_{\nu-1, k+1}$ и $j_{\nu-1, k+2}$. Для определенности сначала предположим, что $J_{\nu-1}(z)>0$ в открытом интервале. Тогда в окрестности точки $j_{\nu-1, k+1}$ имеем неравенство $J_{\nu-1}^{\prime}(z)>0$. Кроме того, $(z+(\nu-1)) / z>0$. Таким образом, второй член в правой части уравнения (22) всегда положителелен. Что же касается первого члена, он тоже положителен при $j_{\nu-1, k+2}^{\prime}<z<j_{\nu-1, k+2}$, поскольку $J_{\nu-1}^{\prime}(z)<0$. Как следствие, сумма $J_{\nu-1}(z)+J_{\nu}(z)$ остается положительной в этом интервале, и поэтому здесь нулей нет.

Далее, при $j_{\nu-1, k+1}<z<j_{\nu-1, k+2}^{\prime}$ второй член в правой части уравнения (22) по-прежнему положителен, но первый член становится отрицательным. Кроме того, $J_{\nu-1}\left(j_{\nu-1, k+1}\right)+J_{\nu}\left(j_{\nu-1, k+1}\right)<0$. Согласно пункту 3 настоящей теоремы существует в точности один корень разности между $j_{\nu-1, k+1}$ и $j_{\nu-1, k+2}$ и он таков, что $j_{\nu-1, k+1}<\lambda_{\nu-1, k+1}^{+}<j_{\nu-1, k+2}^{\prime}<j_{\nu-1, k+2}$ согласно соотношениям (25). Легко проверить, что этот вывод можно получить на основе предположения о том, что $J_{\nu-1}(z)<0$ в открытом интервале.

Наконец, аналогичный анализ знаков суммы $J_{\nu-1}(z)+J_{\nu}(z)$ с помощью уравнения (21) приводит к результату, содержащемуся в соотношениях (24). Утверждение и теорема доказаны. 
СледСтвиЕ 4.1. Справедливы следующие неравенства:

$$
\lambda_{\nu-1, k+1}^{+}<\lambda_{\nu-2, k+2}^{+}<\lambda_{\nu-1, k+2}^{+}
$$

ДокАЗАтЕЛЬство. Эти соотношения получаются непосредственно с помощью подстановки второго из неравенств в цепочке неравенств (25) в выражение (24) после замены индексов $\nu$ на $\nu-1$ и $k$ на $k+1$ и использования первого неравенства в $(25)$ после соответствующей замены индексов.

СлЕДСТВиЕ 4.2. Справедливы неравенства

$$
j_{\nu, k}<\lambda_{\nu-1, k+1}^{-}<j_{\nu, k+1}^{\prime}<\lambda_{\nu-1, k+1}^{+}<j_{\nu, k+1} .
$$

ДокАЗАТЕльство. Доказательство этого следствия аналогично доказательству следствия 4.1, если использовать неравенства (18) и (24).

СледСтвиЕ 4.3. Между двумя последовательными нулями разности $J_{\nu-1}(z)-$ $J_{\nu}(z)$ есть один нуль суммы $J_{\nu-1}(z)+J_{\nu}(z)$, и наоборот.

ДокАзАтЕЛьСтво. Справедливость этого утверждения доказывается итерацией неравенств (29).

СлЕДСтвиЕ 4.4. Справедливы следующие неравенства:

$$
j_{\nu-1, k}<\lambda_{\nu-1, k}^{+}<j_{\nu-1, k+1}^{\prime}<\lambda_{\nu-1, k+1}^{-}<j_{\nu-1, k+1} .
$$

ДокАзАтЕЛьство. Эти неравенства можно получить непосредственно на основе неравенств (17) и (25).

СлЕДСТВИЕ 4.5. Нули производных функций Бесселя первого рода чередуются согласно условию

$$
j_{\nu, k+1}^{\prime}<j_{\nu-1, k+2}^{\prime}<j_{\nu, k+2}^{\prime} .
$$

ДокАзАТЕльство. Справедливость этого утверждения следует непосредственно из неравенств (29) и (30).

\section{5. СРЕДНИЙ ЗАРЯД}

Чтобы получить средний заряд при нулевой температуре в присутствии запирающего потенциала (химического потенциала) $\mu$ с помощью теоретико-полевого метода, нужна функция Грина $G$ оператора Дирака уравнения (1), производная которой по времени смещена на $-i \mu$. С этой целью, как и в работе [28], сначала введем функцию Грина $\mathcal{G}$ вспомогательного оператора $\mathcal{D}=\gamma^{0} \not D$. Легко показать, что $G=\mathcal{G} \gamma^{0}$. В свою очередь $\mathcal{G}$ имеет вид

$$
\mathcal{G}\left(x, x^{\prime}\right)=\sum_{q, \alpha} \int d k_{0} \frac{\psi_{q}(\vec{x}) e^{-i k_{0} x^{0}} \otimes \psi_{q}^{\dagger}\left(\vec{x}^{\prime}\right) e^{i k_{0} x^{\prime 0}}}{k_{0}+\mu+\alpha E_{q}}
$$

где параметр $\alpha= \pm 1$ определяется, как и в разделе $2, \psi_{q}(\vec{x})$ - собственные функции $(9)$, соответствующие $|\mathcal{E}|=E_{q} / v_{\mathrm{F}}$, а $q$ означает порядок появления собственных значений в уравнении (8) с ростом абсолютной величины энергии. 
Хорошо известно [30], что локальное среднее число носителей $n(\mu, x)$ можно представить следующим образом:

$$
n(\mu, x)=-i \operatorname{tr}\left(\gamma^{0} G(x, x)\right)=-i \operatorname{tr}(\mathcal{G}(x, x)) .
$$

С помощью уравнений (9) и (31) получим из выражения (32) среднее число носителей заряда в квантовой наноточке в виде

$$
\begin{aligned}
n(\mu) \equiv \int_{0}^{R} r d r \int_{0}^{2 \pi} d \theta n(\mu, x) & =-i \sum_{\alpha, q} \int_{-\infty}^{\infty} \frac{d k_{0}}{2 \pi} \frac{1}{k_{0}+\mu+\alpha E_{q}}= \\
& =-i \sum_{q} \int_{-\infty}^{\infty} \frac{d k_{0}}{2 \pi} \frac{2\left(k_{0}+\mu\right)}{\left(k_{0}+\mu\right)^{2}-E_{q}{ }^{2}} .
\end{aligned}
$$

Очевидно, что последнее выражение требует ультрафиолетовой регуляризации. Как и в работе [28], будем придерживаться идей, изложенных в работе [30]. Сначала произведем сдвиг $k_{0} \rightarrow k_{0}+i \epsilon \operatorname{sgn}\left(k_{0}\right)$ в знаменателе, что соответствует правилу Фейнмана обращения с полюсами на действительной оси в интеграле (33). Таким образом, имеем

$$
n(\mu)=-i \sum_{q} \int_{-\infty}^{\infty} \frac{d k_{0}}{2 \pi} \frac{2\left(k_{0}+\mu\right)}{\left(k_{0}+i \epsilon \operatorname{sgn}\left(k_{0}\right)+\mu\right)^{2}-E_{q}{ }^{2}} .
$$

Используя теперь новую переменную интегрирования $t=k_{0}+\mu$, получим

$$
n(\mu)=-i \sum_{q} \int_{-\infty}^{\infty} \frac{d t}{2 \pi} \frac{2 t}{(t+i \epsilon \operatorname{sgn}(t-\mu))^{2}-E_{q}{ }^{2}},
$$

или, аналогично,

$$
\begin{aligned}
n(\mu)= & -\frac{i}{2} \sum_{q}\left\{\int_{-\infty}^{\infty} \frac{d t}{2 \pi} \frac{2 t}{(t+i \epsilon \operatorname{sgn}(t-\mu))^{2}-E_{q}{ }^{2}}-\right. \\
& \left.-\int_{-\infty}^{\infty} \frac{d t}{2 \pi} \frac{2 t}{(t+i \epsilon \operatorname{sgn}(t+\mu))^{2}-E_{q}^{2}}\right\}
\end{aligned}
$$

что приводит к

$$
\begin{aligned}
n(\mu) & =\frac{1}{\pi} \sum_{q} \int_{-\infty}^{\infty} d t \frac{t^{2} \epsilon(\operatorname{sgn}(t+\mu)-\operatorname{sgn}(t-\mu))}{\left((t+i \epsilon \operatorname{sgn}(t-\mu))^{2}-E_{q}{ }^{2}\right)\left((t+i \epsilon \operatorname{sgn}(t+\mu))^{2}-E_{q}{ }^{2}\right)}= \\
& =\frac{2}{\pi} \sum_{q} \int_{-|\mu|}^{|\mu|} d t \frac{\epsilon t^{2}}{\left((t+i \epsilon \operatorname{sgn}(t-\mu))^{2}-E_{q}{ }^{2}\right)\left((t+i \epsilon \operatorname{sgn}(t+\mu))^{2}-E_{q}{ }^{2}\right)} .
\end{aligned}
$$

Записывая теперь подынтегральное выражение в виде простых дробей и принимая во внимание, что при $\epsilon \rightarrow 0^{+}$справедливо соотношение $1 /(x \pm i \epsilon)=\mathrm{P} . \mathrm{V} .(1 / x) \mp i \pi \delta(x)$, получим для одной долины и одного спина выражение

$$
n(\mu)=\operatorname{sgn}(\mu) \sum_{q} \Theta\left(|\mu|-\left|E_{q}\right|\right) .
$$




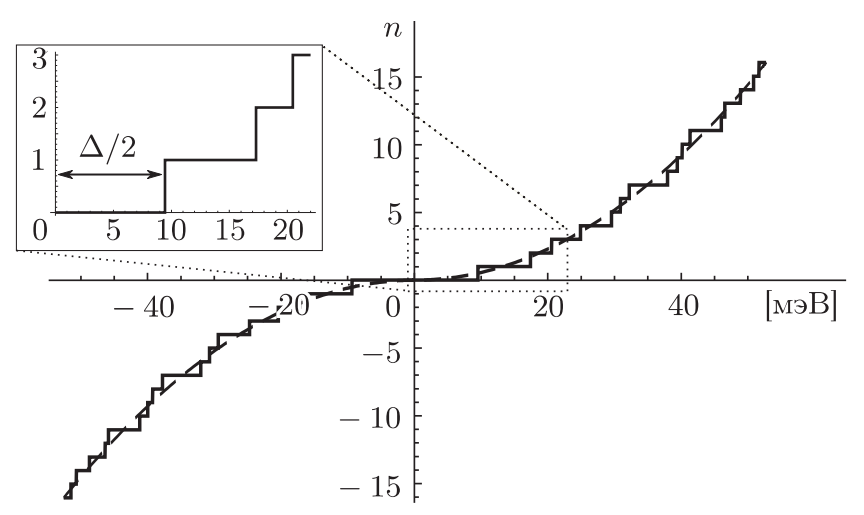

Рис. 1. Сплошная линия - число носителей заряда без учета спинового и долинного вырождения как функция химического потенциала $\mu$ [мэВ] (положительные/отрицательные значения соответствуют числу электронов/дырок) для точки радиуса $R=100$ нм. Штриховая линия - асимптотическое приближение $\langle N\rangle$, представленное уравнением (35).

Этот результат ожидаем, поскольку изучаемое многообразие компактно, и поэтому спектр, определяемый уравнением (8), дискретен. Таким образом, уравнение (34) подтверждает, что для электронов (дырок) каждое заполненное состояние увеличивает (уменьшает) на 1 среднее число.

Хотя фактическое определение точного значения запирающего потенциала, при котором возникают скачки, в случае графенового диска требует численных расчетов, математические результаты, полученные в двух предыдущих разделах, налагают ограничения на число уравнений, которое следует рассматривать в системе (7) при выбранном интервале значений параметра $\mu$. Заметим, что результаты, полученные в двух предыдущих разделах, также гарантируют, что в конечном интервале изменения значений запирающего потенциала средний заряд $n(\mu)$ испытывает конечное число скачков одинаковой высоты, поскольку спектр не вырожден. Это следует непосредственно из правила чередования нулей функций Бесселя, кратность которых равна единице.

Здесь следует напомнить читателю, что средний заряд рассматриваемой наноточки равен произведению $n(\mu)$ на заряд электрона. В правой части уравнения $(34)$ мы видим функцию $N(\mu)=\sum_{q} \Theta\left(|\mu|-\left|E_{q}\right|\right)$ от числа положительных собственных чисел. Воспользовавшись результатом работы [11], можно показать, что асимптотика для сглаженной версии этой ступенчатой функции дается выражением

$$
\langle N(\mu)\rangle \simeq \frac{A\left(\mu / v_{\mathrm{F}}\right)^{2}}{4 \pi}+\cdots,
$$

где $A=\pi R^{2}$ - площадь квантовой точки. Заметим, что такое сглаживание является результатом полуклассического приближения уравнения (8) непрерывным спектром при нулевой температуре [11], [32].

Зависимость числа носителей заряда квантовой точки радиуса $R=100$ нм от значений параметра $\mu$ показана на рис. 1 сплошной линией, зависимость, представленная асимптотическим приближением (35), - штриховой линией. Заметим, что 
результаты разделов 3 и 4 гарантируют отсутствие других скачков внутри рассматриваемого интервала. Результаты, полученные в этих разделах, также показывают, что значение массовой щели $\Delta$ определяется выражением

$$
\Delta=2 \frac{\hbar c v_{\mathrm{F}}}{R} \lambda_{0,1}^{-} \approx 2.8 \frac{\hbar c v_{\mathrm{F}}}{R},
$$

которое при $R=100$ нм дает приближение $\Delta \approx 18.4$ мэВ. Здесь мы использовали константы $\hbar$ и $c$ для более точного сравнения полученного результата с экспериментальными данными. Этот результат качественно согласуется как с экспериментальными, так и с теоретическими исследованиями [29], [32], [33].

Расстояния между последовательными собственными числами $E_{q}$, как и длина ступеней в $n(\mu)$, связаны с шириной кулоновского ромба (Coulomb diamond) при измерениях переноса в наноточках. Мы проверили, что статистическое распределение этих расстояний, рассчитанное по первым 2496 энергетическим уровням, согласуется с распределением Пуассона [11]. Как показано в работе [29], это распределение также согласуется с экспериментальными данными для наноточек промежуточных размеров. K сожалению, в экспериментах еще не проводилось сравнение самих ступеней, изменение длины которых имеет своеобразный повторяющийся характер вдоль всего интервала значений $\mu$. Это сравнение сыграло бы решающую роль для определения эффективности рассмотренной модели при описании графеновых квантовых точек, а также помогло бы объяснить некоторые экспериментальные особенности, зависящие от фактического числа носителей заряда в квантовой точке [32].

Среднее число носителей заряда при конечной температуре можно получить, применив поворот Вика в евклидовом пространстве-времени и заменяя интеграл по $k_{0}$ в уравнении (33) на сумму по частотам Мацубары $(2 l+1) \pi / \beta$, где в натуральных единицах $\beta=1 / T, T$ - температура. Непосредственные расчеты приводят к следующему результату:

$$
n(\mu)=\operatorname{sgn}(\mu) \sum_{q}\left(\left(\left(1+e^{\left(\left|E_{q}\right|-|\mu|\right) \beta}\right)^{-1}-\left(1+e^{\left(\left|E_{q}\right|+|\mu|\right) \beta}\right)^{-1}\right) .\right.
$$

Этот результат относится к числу тех, которые, как ожидается, можно получить на основе распределения Ферми-Дирака. Он сводится к выражению (34) в пределе нулевой температуры $\beta \rightarrow \infty$. С ростом температуры ступени становятся все более гладкими.

\section{6. НЕКОТОРЫЕ КОММЕНТАРИИ И ЗАМЕЧАНИЯ}

Основные математические результаты статьи содержатся в теоремах $3.1,4.1$ и их следствиях. Установлено точное расположение нулей сумм и разностей функций Бесселя первого рода с различающимися на единицу порядками в том случае, когда эти порядки - действительные неотрицательные числа, а также взаимное чередование этих нулей между собой, с нулями функций Бесселя того же рода и с нулями их производных. Мы также получили новое доказательство правила чередования нулей производных функций Бесселя первого рода. Другие доказательства этого свойства представлены совсем недавно в работах [7]-[9]. Хотя в нашей физической задаче используются только функции Бесселя целого порядка, полученные результаты являются более общими и остаются справедливыми для всех действительных 
неотрицательных порядков. Заметим, что некоторые из этих результатов можно также получить другим способом, воспользовавшись утверждениями леммы 4 из работы [9]. Например, если пункт 2 теоремы 3.1 считать доказанным, утверждение пункта 3 этой теоремы, как нетрудно показать, следует из свойства транзитивности, о котором говорится в данной лемме.

В качестве физического обоснования нашей математической задачи мы изучили спектр графеновых наноточек с геометрией диска, а также вычислили их средний заряд и его зависимость от внешнего запирающего напряжения (химического потенциала). Эта зависимость была найдена в разделе 5 и наглядно представлена на рис. 1. Хотя фактическое определение точного значения запирающего потенциала, при котором возникают скачки, требует численных расчетов, математический результат, подробно рассмотренный в предыдущем разделе, налагает ограничение на число уравнений, которое нужно учесть в системе (7) при выбранном интервале значений $\mu$. Заметим, что, как и следовало ожидать для конечного образца, в квантовой проводимости графенового диска $e^{2} \partial n / \partial \mu$ возникают последовательные пики, появляющиеся всякий раз при переходе запирающего напряжения через разрешенное значение энергии. В рассматриваемой нами геометрии и при выбранных ГУ эти пики расположены на различных расстояниях друг от друга. Экспериментальное изучение графеновых наноточек находится все еще на самой предварительной ступени и в основном посвящено исследованию свойств переноса [18], но некоторые свидетельства в пользу рассмотренной модели уже очевидны [29].

В случае ГУ Берри-Мондрагона можно с уверенностью утверждать, что радиальный ток, входящий в точку или выходящий из нее, не разрешен. То же справедливо для любых локальных ГУ, приводящих к самосопряженному гамильтониану, к которым относятся и зигзагообразные граничные условия [13]. Однако существование тангенциального тока, запрещенного в зигзагообразном случае, не запрещено ГУ Берри-Мондрагона. Свойства этого тока и возможность его использования в электронных приборах заслуживают изучения.

Наконец, стоит упомянуть, что, как указано в работе [34], наши теоремы также полезны при изучении теорий поля в ограниченных областях большей размерности с цилиндрической симметрией.

\section{Список литературы}

[1] Р. Курант, Д. Гильберт, Методы математической физики, т. 1, Гостехиздат, М.-Л., 1933.

[2] G. N. Watson, A Treatise on the Theory of Bessel Functions, Cambridge Univ. Press, Cambridge, 1944.

[3] E. K. Ifantis, P. D. Siafarikas, J. Comput. Appl. Math., 21:2 (1988), 245-249.

[4] E. K. Ifantis, C. G. Kokologiannaki, C. B. Kouris, J. Comput. Appl. Math., 34:1 (1991), $21-31$.

[5] Á. Elbert, J. Comput. Appl. Math., 133:1-2 (2001), 65-83.

[6] E. N. Petropoulou, P. D. Siafarikas, I. D. Stabolas, J. Comput. Appl. Math., 153:1-2 (2003), 387-393.

[7] H. Liu, J. Zou, Research Report CUHK-2007-02 (342), The Chinese University of Hong Kong, Hong Kong:, 2007; IMA J. Appl. Math., 72:6 (2007), 817-831.

[8] T. Pálmai, B. Apagyi, J. Math. Anal. Appl., 375:1 (2011), 320-322.

[9] T. Pálmai, Math. Inequal. Appl., 16:1 (2013), 241-247. 
[10] G. Esposito, G. Fucci, A. Yu. Kamenshchik, K. Kirsten, JHEP, 09 (2005), 063, 17 pp., arXiv: hep-th/0507264.

[11] M. V. Berry, R. J. Mondragon, Proc. Roy. Soc. London Ser. A, 412:1842 (1987), 53-74.

[12] A. Chodos, R. L. Jaffe, K. Johnson, C. B. Thorn, V. F. Weisskopf, Phys. Rev. D, 9:12 (1974), 3471-3495.

[13] C. G. Beneventano, E. M. Santangelo, Internat. J. Modern Phys. Conf. Ser., 14 (2012), $240-250$.

[14] В. А. Волков, И. В. Загороднев, ФНТ, 35:1 (2009), 5-9.

[15] A. K. Geim, K. S. Novoselov, Nature Mater., 6:3 (2007), 183-191.

[16] A. H. Castro Neto, F. Guinea, N. M. R. Peres, K. S. Novoselov, A. K. Geim, Rev. Modern Phys., 81:1 (2009), 109-162, arXiv: 0709.1163.

[17] S. Das Sarma, Shaffique Adam, E. H. Hwang, Enrico Rossi, Rev. Modern Phys., 83:2 (2011), 407-470, arXiv: 1003.4731.

[18] M. Katsnelson, Graphene: Carbon in Two Dimensions, Cambridge Univ. Press, Cambridge, 2012.

[19] G. W. Semenoff, Phys. Rev. Lett., 53:26 (1984), 2449-2452.

[20] D. P. DiVincenzo, E. J. Mele, Phys. Rev. B, 29:4 (1984), 1685-1694.

[21] K. S. Novoselov, A. K. Geim, S. V. Morozov, D. Jiang, M. I. Katsnelson, I. V. Grigorieva, S. V. Dubonos, A. A. Firsov, Nature, 438:7065 (2005), 197-200, arXiv: cond-mat/0509330.

[22] I. V. Fialkovsky, D. V. Vassilevich, Internat. J. Modern Phys. A, 27:15 (2012), 1260007, 12 pp., arXiv: 1111.3017.

[23] A. Cortijo, F. Guinea, M. A.H. Vozmediano, J. Phys. A: Math. Theor., 45:38 (2012), 383001, 47 pp., arXiv: 1112.2054.

[24] C. G. Beneventano, P. Giacconi, E. M. Santangelo, R. Soldati, J. Phys. A: Math. Theor., 40:24 (2007), F435-F442, arXiv: hep-th/0701095.

[25] S. M.-M. Dubois, Z. Zanolli, X. Declerck, J.-C. Charlier, Eur. Phys. J. B, 72 (2009), 1-24.

[26] C. Stampfer, S. Fringes, J. Güttinger, F. Molitor, C. Volk, B. Terrés, J. Dauber, S. Engels, S. Schnez, A. Jacobsen, S. Dröscher, T. Ihn, K. Ensslin, Front. Phys., 6:3 (2011), 271-293.

[27] I. Snook, A. Barnard, "Graphene nano-flakes and nano-dots: Theory, experiment and applications", Physics and Applications of Graphene - Theory, ed. S. Mikhailov, InTech, Rijeka, Croatia, 2011, 277-302.

[28] C. G. Beneventano, I. V. Fialkovsky, E. M. Santangelo, D. V. Vassilevich, Eur. Phys. J. B, 87:3 (2014), 50, 9 pp., arXiv: 1311.0254.

[29] L. A. Ponomarenko, F. Schedin, M. I. Katsnelson, R. Yang, E. W. Hill, K. S. Novoselov, A. K. Geim, Science, 320:5874 (2008), 356-358, arXiv: 0801.0160.

[30] A. Chodos, K. Everding, D. A. Owen, Phys. Rev. D, 42:8 (1990), 2881-2892.

[31] H. P. Baltes, E. R. Hilf, Spectra of finite systems. A Review of Weyl's Problem: The Eigenvalue Distribution of the Wave Equation for Finite Domains and Its Applications on the Physics of Small Systems, Bibliographisches Institut, Mannheim-Vienna-Zurich, 1976.

[32] S. Schnez, K. Ensslin, M. Sigrist, T. Ihn, Phys. Rev. B, 78:19 (2008), 195427, 4 pp., arXiv: 0810.3216.

[33] S. Schnez, F. Molitor, C. Stampfer, J. Güttinger, I. Shorubalko, T. Ihn, K. Ensslin, Appl. Phys. Lett., 94:1 (2009), 012107, 3 pp., arXiv: 0807.2710.

[34] V.E. Ambrus, Dirac fermions on rotating space-times, PhD Thesis, University of Sheffield, Sheffield, UK, 2014. 